\title{
The Influence of Energetic Factors on Biomarkers of Postmenopausal Breast Cancer Risk
}

\author{
Heather K. Neilson • Shannon M. Conroy • \\ Christine M. Friedenreich
}

Published online: 15 December 2013

(C) The Author(s) 2013. This article is published with open access at Springerlink.com

\begin{abstract}
Strong and consistent evidence exists that physical activity reduces breast cancer risk by $10-25 \%$, and several proposed biologic mechanisms have now been investigated in randomized, controlled, exercise intervention trials. Leading hypothesized mechanisms relating to postmenopausal breast cancer include adiposity, endogenous sex hormones, insulin resistance, and chronic low-grade inflammation. In addition, other pathways are emerging as potentially important, including those involving oxidative stress and telomere length, global DNA hypomethylation, immune function, and vitamin D exposure. Recent exercise trials in overweight/obese postmenopausal women implicate weight loss as a mechanism whereby exercise induces favorable changes in circulating estradiol levels and other biomarkers as well. Still it is plausible that some exercise-induced biomarker changes do not require loss of body fat, whereas others depend on abdominal fat loss. We highlight the latest findings from randomized, controlled trials of healthy postmenopausal women, relating exercise to proposed biomarkers for postmenopausal breast cancer risk.
\end{abstract}

H. K. Neilson · S. M. Conroy $\cdot$ C. M. Friedenreich $(\bowtie)$

Department of Population Health Research, CancerControl Alberta, Alberta Health Services, Quarry Park, c/o 10101 Southport Rd SW, Calgary, Alberta T2W 3N2, Canada

e-mail: Christine.Friedenreich@albertahealthservices.ca

H. K. Neilson

e-mail: Heather.Neilson@albertahealthservices.ca

S. M. Conroy

e-mail: Shannon.Conroy@albertahealthservices.ca

C. M. Friedenreich

Department of Community Health Sciences, Faculty of Medicine, University of Calgary, 3330 Hospital Drive N.W., Calgary,

Alberta T2N 4N2, Canada

C. M. Friedenreich

Department of Oncology, Faculty of Medicine, University of

Calgary, 133129 St. N.W., Calgary, Alberta T2N 4N2, Canada
Keywords Breast cancer · Postmenopausal women · Randomized trials $\cdot$ Physical activity $\cdot$ Exercise $\cdot$ Weight loss . Adiposity $\cdot$ Biomarkers $\cdot$ Sex hormones $\cdot$ Estrogen . Biomechanisms

\section{Introduction}

There is convincing epidemiologic evidence that body fatness, and probable evidence that adult weight gain, are associated with an increased risk of postmenopausal breast cancer [1]. One meta-analysis demonstrated that every $5 \mathrm{~kg} / \mathrm{m}^{2}$ increase in body mass index (BMI) increases postmenopausal breast cancer risk by $12 \%$ on average [2] with possible variation by tumor subtype [3]. In addition, after a breast cancer diagnosis, survival rates are decreased with higher BMI [4] by as much as $30 \%$ [5].

It is of great interest, therefore, to prospectively study the effects of weight control on breast cancer risk and survival in overweight or obese postmenopausal women [6]. Yet first, understanding the contributions of energetic factors-i.e., physical activity and diet — is needed to determine the optimal weight control intervention. Regular physical activity is a widely accepted health-promoting behavior that is recommended for cancer prevention [1,7], but the type and dose of activity that is optimal for postmenopausal breast cancer prevention remains unclear.

Physical activity and breast cancer

Convincing epidemiologic evidence suggests that physical activity of moderate-to-vigorous intensity reduces breast cancer risk by $10-25 \%$ on average relative to inactivity $[8 \cdot, 9]$ The dose of activity required for breast cancer prevention is unclear, but across observational studies, risk generally decreases with higher physical activity duration [8•] and 
intensity [9]. For cancer prevention overall, public health recommendations advise at least 30 minutes of moderateintensity activity equivalent to brisk walking every day [1] or 30 minutes or more of moderate-to-vigorous activity at least 5 days per week [7] for adults.

\section{Biomarker studies in healthy women}

Given clear inverse relations between physical activity and future postmenopausal breast cancer risk, lifestyle modification for inactive women holds promise for breast cancer prevention. Whereas randomized, controlled trials (RCTs) examining breast cancer outcomes would best inform an exercise prescription, these trials have not been conducted because of the large sample size and time required for a prospective study. A more feasible approach is to study the impact of lifestyle change on breast cancer biomarkers using a RCT [10]. The number of exercise RCTs studying proposed biomarkers for breast cancer has escalated during the past 10 years, shedding light on: 1) exercise prescriptions that might impact breast cancer risk, and 2) underlying biologic mechanisms.

We provide an update on the epidemiologic evidence relating exercise to proposed biomarkers for postmenopausal breast cancer risk, without dietary modification. This review enhances our earlier reviews $[8,11]$ by focusing on the strongest, most up-to-date epidemiologic evidence (from randomized trials) relating exercise to estrogens and adiposity, the two most convincing biomarkers of postmenopausal breast cancer risk. We also update our biologic model that relates physical activity to breast cancer by incorporating newly hypothesized biomarkers. Our goal is to guide future clinical and mechanistic research surrounding physical activity and postmenopausal breast cancer prevention. The focus of this review will be on healthy women in whom biomarker profiles and the types and effects of prescribed exercise may differ from breast cancer survivors. Biomarker studies in breast cancer survivors are reviewed elsewhere $[12 \bullet, 13]$.

\section{Epidemiologic evidence relating exercise to proposed biomarkers}

To simplify our discussion of the existing epidemiologic evidence, we have classified proposed biomarkers of breast cancer risk as "convincing" or "hypothesized." Our classification of estrogens and adiposity as convincing biomarkers is based on the relatively strong and consistent body of epidemiologic evidence relating these markers to postmenopausal breast cancer risk. We summarize that evidence and describe in more detail the effects of exercise on estrogen levels (by systematically reviewing RCT evidence) and adiposity (citing recent reviews and large RCTs), specifically in postmenopausal women. We then provide a high-level overview of other, hypothesized biomarkers of postmenopausal breast cancer risk and possible relations with exercise.

Systematic review of RCTs relating exercise to estrogens

In September 2013, we searched the published literature (PubMed-NIH) for RCTs that studied the impact of exercise on estrogens. In brief, we identified all RCTs of long-term exercise ( $\geq 4$ weeks) that compared exercise-only to a nonexercise control group in healthy postmenopausal women. Studies exclusive to hormone replacement therapy users were excluded as were studies in morbidly obese women (BMI $>40 \mathrm{~kg} / \mathrm{m}^{2}$ ) and trained athletes.

Convincing biomarkers for postmenopausal breast cancer risk

\section{Adiposity}

Body fatness is an accepted, convincing biomarker for increased postmenopausal breast cancer risk in healthy women $[1,2]$. Multiple interrelated biologic pathways could mediate the association between adiposity and postmenopausal breast cancer, with sex hormones, insulin resistance, and low-grade chronic inflammation as leading hypotheses $[14 \bullet, 15]$. Furthermore, central adiposity may be particularly important. Recently in postmenopausal women, independently of BMI, waist circumference was positively associated with breast cancer risk [16] and abdominal fat was related to sex hormone bioavailability [17•], which is a strong biomarker of breast cancer risk. Therefore, with respect to postmenopausal breast cancer, there may be more benefit from exercise prescriptions that can effectively lower abdominal fat.

Exercise is publicly recommended for modest weight loss, for prevention of weight gain in overweight and obese adults, and for prevention of weight regain after weight loss $[18,19]$. Exercise trials typically produce $<3 \%$ weight loss in adults, although more might be achieved with higher volumes of exercise, e.g., the American College of Sports Medicine recently proposed $>250$ minutes per week at moderate intensity [18]. However exercise-induced weight loss could vary by age. In a prospective, observational study of 58,610 postmenopausal women, whether body weight was lost, maintained, or gained with high levels of physical activity generally depended on the age of the women at baseline [20]. Also, exercise type could be relevant, e.g., aerobic may be preferable to resistance exercise with respect to weight loss [18] and lowering total abdominal fat [21•] in overweight adults. Indeed, the largest exercise RCTs of moderate-vigorous aerobic exercise in healthy postmenopausal women all showed decreases in intra-abdominal fat $[22,23]$ or waist circumference $[24 \bullet, 25$, $26]$ and overall body fat [22, 23, 24•, 25]. 


\section{Sex hormones}

Higher levels of endogenous estrogens and androgens and lower levels of circulating sex hormone binding globulin (SHBG) are related to an increased risk of postmenopausal breast cancer [27]. In addition, associations between postmenopausal breast cancer risk and hormone replacement therapy use [28] and effective use of antiestrogenic drugs to prevent breast cancer [29] firmly support a causal role for estrogens. Estrogens can decrease apoptosis and act as mitogens in the breast via estrogen receptor binding; moreover, oxidative estrogen metabolites act as mutagenic, genotoxic agents possibly contributing to breast cancer initiation [30].

A total of nine exercise-only RCTs in postmenopausal women that studied changes in estrogen-related biomarkers for breast cancer were identified in our systematic review of published literature (Table 1). The number of non-HRT users assigned to exercise-only or control groups ranged from 16 [31] to 320 [32]. All study populations were overweight or obese on average, and all but one RCT of women 65+ years [33] studied younger postmenopausal women with mean ages from 54-61 years. Roughly half of the trials, comprising the four largest RCTs [32, 34, 35, 36••] and one smaller RCT [37], involved 12-month interventions, whereas the remainder were $12[31,33]$ or $16[38,39]$ weeks duration. Most interventions were aerobic $[32,33,35,36 \bullet, 38]$ or combined aerobic/ resistance training [34, 37]; two small RCTs focused on resistance training $[31,39]$. Exercise prescriptions ranged from 150-225 minutes/week (except [31] where minutes per week were not reported) and were generally moderate-vigorous intensity (i.e., 60-85 \% maximum heart rate).

Table 2 summarizes results for estradiol, the most biologically potent estrogen [15], and also for estrone and SHBG from RCTs in Table 1. Findings on other estrogen-related biomarkers are described below. Nearly all of the reports in our systematic review described circulating hormone levels [31-35, 36••, 37-39] with single articles describing analysis of urine [40] and adipose tissue [41]. Results across RCTs were remarkably consistent, generally showing average decreases in sex hormones and increases in SHBG levels in exercise groups, typically $<10 \%$ in magnitude. Yet only a few primary analyses demonstrated statistically significant differences between exercise and control groups with respect to change in total estradiol [32], free estradiol [32, 35], estrone $[35,36 \bullet \cdot]$, or SHBG $[32,38]$. No statistically significant group differences were found in primary analyses of testosterone or androstenedione [31-34, 36••, 37, 39, 42]. In the SHAPE trial, however, a significant intervention effect was found for testosterone and androstenedione (decreased levels in exercisers versus controls) for the subgroup who lost $>2 \%$ body fat [34]. The Physical Activity for Total Health study [40] showed no significant differences after 12 months between exercise $(\mathrm{n}=$ $87)$ and control groups $(n=86)$ for changes in 2- hydroxyestrone, $16 \alpha$-hydroxyestrone, or their ratio in urine. In an ancillary study of the NEW trial $(n=45)$ [41], subcutaneous adipose tissue was analyzed for expression of 82 candidate genes related to adipokines, proinflammatory cytokines, and sex hormones. Combining women from all four trial arms, greater weight loss after 6 months was associated with decreased gene expression related to estrogen biosynthesis, e.g., 17ß-hydroxysteriod dehydrogenase 1 , which converts estrone to estradiol.

A mediating role for weight loss in the causal pathway between exercise and decreased estrogen levels is plausible given that adipose tissue is the primary source of endogenous estrogens post-menopause [15]. Evidence of mediation by adiposity change, described in Table 2 , generally supports this hypothesis. Moreover, in the NEW trial, substantially greater decreases in total and free estradiol levels occurred, on average, within the diet+exercise arm than with exercise-only ( $-20.3 \%$ vs. $-4.9 \%$ for estradiol; $-26 \%$ vs. $-4.7 \%$ for free estradiol) [36•*]. In a smaller weight loss trial of obese postmenopausal women, a $14 \%$ average decrease in total fat mass was associated with a $24 \%$ decrease in estradiol levels in breast ductal fluid [43•]. In a recent prospective study, a $12.7 \%$ decrease in total estradiol was estimated for every $1 \mathrm{~kg} / \mathrm{m}^{2}$ decrease in BMI in 84 postmenopausal women who lost weight [44]. Interestingly in another recent study of 1,180 postmenopausal women [17•], waist circumference and waistto-hip ratio - independently of BMI - were associated with circulating SHBG, free estradiol, and free testosterone levels, implicating abdominal fat as a specific target for breast cancer prevention.

Hypothesized biomarkers of postmenopausal breast cancer risk

\section{Insulin resistance}

Insulin resistance, characterized by hyperinsulinemia, is a major predictor of diabetes risk and of possible etiologic importance in breast cancer $[45,46]$. Insulin receptor binding promotes mitosis and antiapoptotic effects in breast cancer cells, and also tumor cell migration and tumor-associated angiogenesis [47]. In addition, chronically elevated insulin can enhance estrogen bioactivity and promote activities of breast cancer-related adipokines [47] and IGF-1 [14•]. Metformin, a pharmacologic agent that improves insulin sensitivity, is undergoing clinical testing for improved breast cancer survival $[14 \cdot, 48]$.

In the United States, at least 150 minutes per week of moderate-vigorous aerobic and resistance exercise is recommended for diabetes prevention in prediabetics [49]. In larger RCTs of postmenopausal women, long-term aerobic exercise groups experienced average decreases of approximately $-4 \%$ to $-10.3 \%$ in insulin $[50,51,52 \cdot, 53],-2 \%$ to $-11.4 \%$ in 


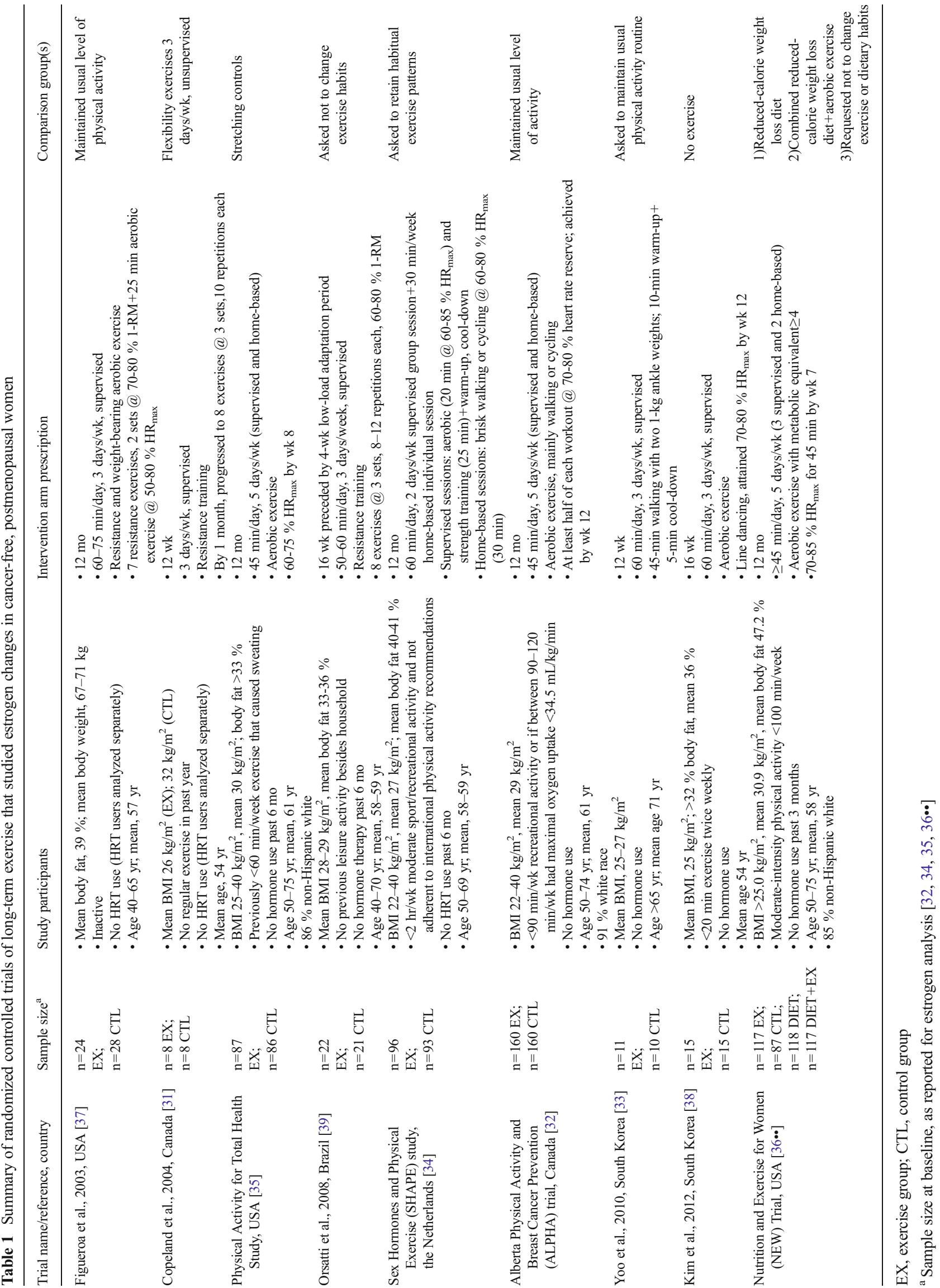







homeostatic model of assessment for insulin resistance (HOMA-IR) [50, 51, 52•], $-3.7 \%$ in C-peptide [52•], and a small decrease [53] or negligible change in circulating glucose $[50,51,52 \bullet]$. However, in the NEW trial [52•], HOMA-IR decreased an average of $-24 \%$ and $-26 \%$ in the diet and diet + exercise intervention groups, respectively, after 12 months, suggesting strongly that improved whole-body insulin sensitivity in the exercise-only group $(-8.6 \%$ decrease in HOMA-IR) resulted from weight loss and not exercise per se. Secondary analyses of ALPHA trial data [54•] implied partial mediation by change in intra-abdominal fat area, which is of etiologic relevance to insulin resistance [55], and by total (\%) body fat but also mediation by other unidentified factors as well.

Changes in insulin sensitivity could vary with different exercise prescriptions. In large RCTs of postmenopausal women, the greatest improvement in HOMA-IR was found with aerobic exercise $>225$ minutes/week [50] and $>130$ minutes/week [51]. In the NEW trial, 225 minutes/ week of prescribed aerobic exercise was associated with a regression to normal fasting glucose levels for those with impaired glucose tolerance at baseline [52•]. Resistance training could provide distinct benefits for glycemic control by altering the quality and quantity of skeletal muscle [56]. However, in an RCT comparing 8 months of aerobic versus resistance exercise in 155 overweight adults [21•], only aerobic exercise reduced HOMA and visceral fat. Similarly a RCT of obese postmenopausal women showed essentially no change in average insulin levels after 12 weeks of lowintensity resistance training [57].

\section{Adipokines}

Adipose tissue is an active endocrine organ, secreting bioactive factors known as adipokines [58] at abnormal levels in obesity. Some adipokines (TNF- $\alpha$, IL-6) are mediators of a low-grade, systemic inflammatory state that is characteristic of obesity [59]. Adipokines, such as leptin, TNF- $\alpha$, and IL-6, could mediate breast cancer development and progression directly by acting as mitogens in the breast, inhibiting apoptosis, and influencing tumor cell migration and invasion [15, 60]. They also may act indirectly, e.g., by enhancing estrogen bioactivity and promoting insulin resistance [15]. Conversely, adiponectin is an adipokine that occurs at lower levels in obesity and is anti-mitogenic, anti-inflammatory, and promotes insulin sensitivity. Epidemiologic findings relating adipokines in circulation to increased postmenopausal breast cancer risk are generally mixed, but suggestive, for increased leptin [61-63], decreased adiponectin [62-64], and a decreased adiponectin:leptin ratio [62]. There is weaker epidemiologic evidence of etiologic roles for IL-6 [65], TNF- $\alpha$ [63, 66], and the inflammatory marker C-reactive protein (CRP) $[62,65]$, produced in response to TNF- $\alpha$ and IL-6.
RCT findings overall imply that exercise in conjunction with sufficient weight loss can decrease circulating leptin [50, $51,67 \bullet]$ and perhaps IL-6 [43•,68•] in postmenopausal women. Exercise-related decreases in CRP have been found in some RCTs of postmenopausal women [69-71], but not all [26], and in others, only with concurrent weight loss [68・ 72]. The Dose-response to Exercise in postmenopausal Women (DREW) study compared three doses of aerobic exercise $(4,8$, or $12 \mathrm{kcal} / \mathrm{kg} /$ week at $50 \% \mathrm{VO}_{2 \max }$ ), but revealed no difference with respect to CRP change in exercisers versus controls over 6 months [72]. Six RCTs of postmenopausal women consistently showed no change in adiponectin levels, on average, with exercise alone $[50,53,57,67 \cdot, 69,73]$. Furthermore, in primary analyses from multiple RCTs there was no reported effect of exercise-only on TNF- $\alpha[53,69,70]$ or IL-6 $[53,68 \bullet, 69-71,73]$ levels in postmenopausal women.

Weight loss is a plausible mediator of exercise-induced adipokine changes given strong biologic rationale and a growing body of RCT evidence, particularly for leptin. The NEW trial, for example, demonstrated up to a $53 \%$ average decrease in leptin concentrations in subgroups with $\geq 10 \%$ weight loss, which far exceeded changes in subgroups with $<5 \%$ weight loss (e.g., $5.5 \%$ average decrease, exercise-only) [67•]. The mechanisms driving exercise-related inflammatory marker changes (CRP, TNF- $\alpha$, IL-6) are probably more complex, relating not only to weight loss but potentially also to effects on muscle tissue, endothelial cells, and immune cells [59].

\section{Immune function}

Evading immunological destruction is an emerging hallmark of cancer and a diminished competence of the immune response is a recognized predictor of cancer risk; however, there is currently no consensus on which immune biomarkers are causally related to cancer risk $[74,75]$.

Physical activity might impede carcinogenesis by moderating the innate and adaptive immune systems [76] and, thus, enhance immunosurveillance and the tumor suppression capacity of the immune system. Furthermore, exercise could help to modulate obesity-related, proinflammatory immune responses [59] and prevent age-related immunosenescence [77]. To date, the acute, transient effects of exercise on immune function have been studied extensively, supporting beneficial effects with moderate-intensity but detrimental effects with high-intensity activity [76]. Although some studies have demonstrated altered number and function of circulating immune cells (e.g., enhanced natural killer cell cytotoxicity and T-lymphocyte proliferation capacity) with long-term exercise, there is a lack of supportive evidence from RCTs [76-78]. For example, the 12-month Physical Activity for Total Health study found no effect of aerobic exercise on in vitro natural killer cell cytotoxicity, T-lymphocyte proliferative response to stimulation, or the relative proportion of immune cell counts 
(e.g., T-cells, helper T-cells, cytotoxic T-cells, B cells, natural killer cells) [79].

\section{Oxidative stress and telomere length}

Oxidative stress results from an imbalance of increased systemic reactive oxygen species (ROS) production and/or reduced antioxidant capacity, including the ability to neutralize reactive intermediates and repair subsequent damage. It is hypothesized to play a central role in breast carcinogenesis [80] and in carcinogenic causal pathways linked to obesity [81]. ROS-induced damage to macromolecules leads to genetic alterations [82]. Because telomeres, nucleoprotein repeats at the ends of chromosomes that protect cells from chromosomal instability, suffer disproportionately from oxidative damage, an important etiologic pathway through which oxidative stress might affect breast cancer risk is through telomere attrition [83].

As part of a favourable biological adaptive response, regular exercise enhances antioxidant and oxidative damage repairing enzyme capacity and may subsequently reduce oxidative damage [84]. The epidemiologic evidence supporting that regular physical activity reduces oxidative damage to macromolecules or telomere attrition is, thus far, limited but suggestive. In the Physical Activity for Total Health study, there was decreased oxidative damage to lipids as measured by $\mathrm{F}_{2}$-isoprostane levels in exercisers compared to controls, with a more pronounced statistically significant reduction in exercisers who increased their physical fitness by $>15 \%$; this effect occurred independent of obesity status [85]. RCTs of Tai Chi exercise [86] and resistance training [87] also showed reductions in oxidative damage. Yet with respect to telomere length, the NEW trial found no effect of dietary weight loss and/or aerobic exercise [88•], nor did a diet-physical activity lifestyle intervention RCT for diabetes prevention in high-risk adults [89].

\section{Global DNA hypomethylation}

The methylation of DNA is recognized as a key epigenetic mechanism in the regulation of gene expression and chromosomal stability. Global DNA hypomethylation in peripheral blood leukocytes represents a postulated biomarker for cancer risk [90] and epidemiologic evidence of an association with increased breast cancer risk is accumulating [91, 92]. Potential epigenetic modifications induced by exercise have been described [93•], and, to-date, two observational studies showed positive associations between physical activity and prevalent repetitive sequences (LINE-1) methylation, a surrogate measure of global methylation [94]. In middle-aged, white women with a family history of breast cancer, higher self-reported physical activity $(\geq 9.8,5.9$, and 12.5 hours per week for childhood, teenage years, and past 12 months, respectively) was associated with a favorable $33 \%$ increase of LINE-1 methylation [95]. Similarly, in another study, cancer-free adults with 26-30 minutes per day of recent physical activity (versus $\leq 10$ minutes per day) as measured via accelerometer, had higher LINE-1 methylation [96].

\section{5-hydroxyvitamin D}

A protective, inverse association between vitamin $\mathrm{D}$ exposure and postmenopausal breast cancer risk is becoming increasingly clear [97, 98]. For some individuals, outdoor physical activity could improve vitamin $\mathrm{D}$ status by increasing cutaneous production of vitamin $\mathrm{D}_{3}$ when UV-B exposure is sufficiently high. Another mechanism involves body composition, because the metabolite 25 -hydroxyvitamin $\mathrm{D}(25(\mathrm{OH}) \mathrm{D})$, the most common serum indicator of vitamin D status, might sequester in body fat [99]. Evidence from the NEW trial supports this hypothesis; in overweight/obese postmenopausal women, sufficient weight loss ( $\geq 15 \%$ body weight) over 12 months, whether induced with aerobic exercise or caloric restriction, increased serum $25(\mathrm{OH}) \mathrm{D}$ concentrations significantly relative to controls ( $p$-trend $=0.002)[100 \bullet]$. Furthermore a 2-year weight loss trial of 383 overweight/obese women revealed a clear dose-response relation between increasing weight loss and increasing serum $25(\mathrm{OH}) \mathrm{D}$ concentrations ( $p$-trend $=0.005$ ), and in multivariable models, weight loss $>10 \%$ was identified as a significant predictor of $25(\mathrm{OH}) \mathrm{D}$ change [101].

\section{Proposed Biologic Model}

\section{An updated model}

Figure 1 depicts a proposed, updated $[8 \cdot, 11]$, biologic model relating physical activity to postmenopausal breast cancer risk via interrelated pathways with common linkages to adiposity. Notable exclusions from our model include mammographic density, which is a strong risk factor for breast cancer [102]; however, evidence from observational studies and RCTs overall do not support an association between long-term exercise and breast density [103]. Similarly, while elevated circulating IGF-1 might signify an increased risk for postmenopausal breast cancer [104], it has not been shown to be decreased with physical activity $[50,105,106 \bullet$. Furthermore, we acknowledge that DNA damage, e.g., resulting from oxidative stress or genotoxic estrogen metabolites, could initiate breast cancer and that DNA repair mechanisms might be enhanced with physical activity [10]; however, this topic was beyond the scope of our review.

Role of body fat

Adiposity change could play a mediating role for any of the biomarkers proposed in our model, or for some biomarkers, 


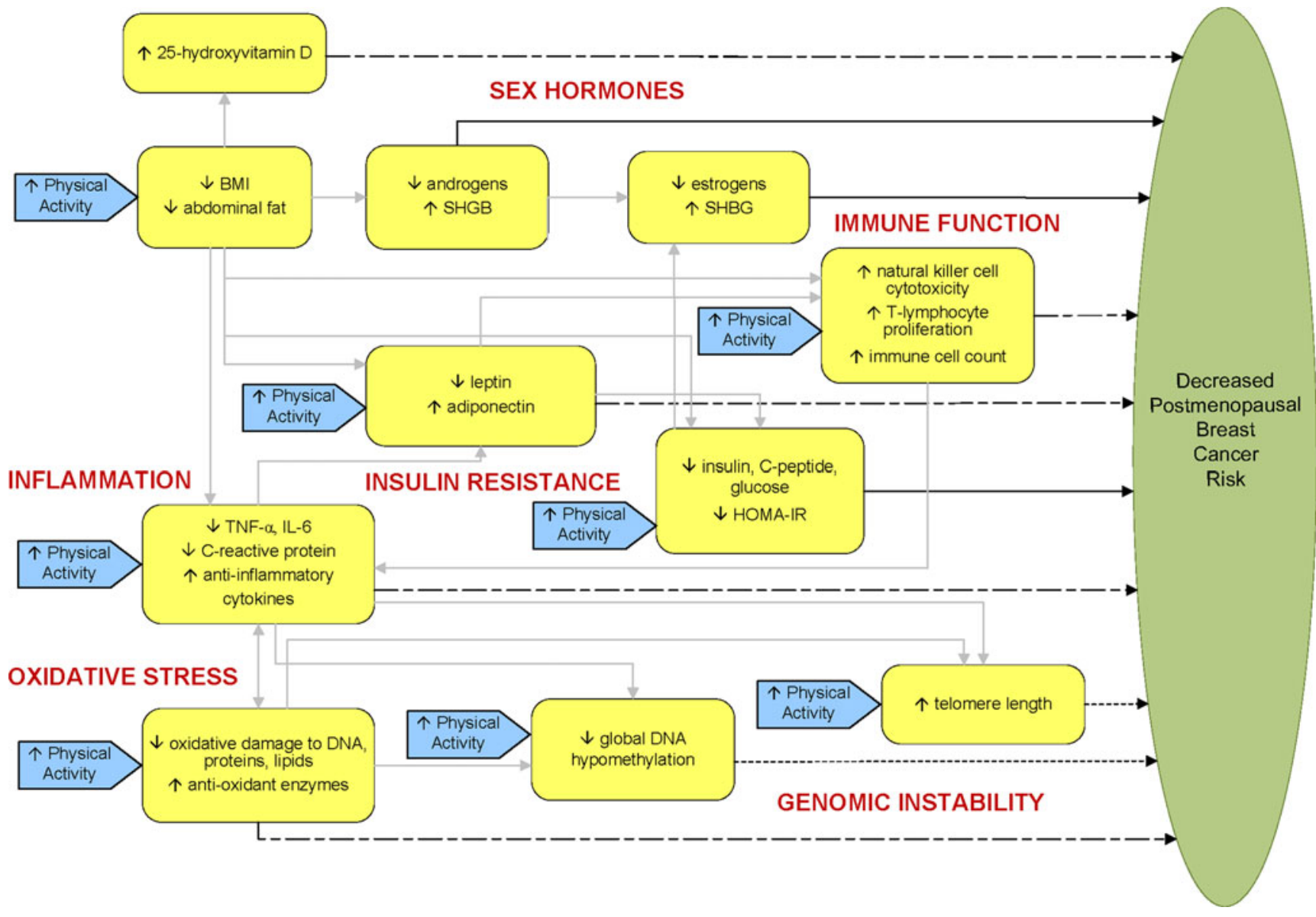

Fig. 1 Hypothesized biological model relating physical activity to postmenopausal breast cancer risk. Strong epidemiologic evidence of an association with breast cancer risk (solid black arrows); limited epidemiologic evidence (irregular dashed arrows ); emerging epidemiologic

physical activity might act independently. The state of being overweight or obese also could modify the effects of physical activity on other biomarkers. Distinguishing the effects of "fat and fit" on breast cancer biomarkers is methodologically challenging and of interest [44, 54•, 107]. Four-armed randomized trials, such as the NEW trial and the recent SHAPE-2 study [108••], were designed to address the fit-versus-fat controversy directly and provide some of the clearest distinctions between exercise and weight loss. Published findings from the NEW trial and other RCTs in this review imply particularly that exercise-related changes in estrogens, SHBG, leptin, and 25-hydroxyvitamin D are mediated largely by weight loss in healthy postmenopausal women.

\section{Research Opportunities}

While considerable advances have been made in our understanding of the biologic mechanisms relating physical activity to postmenopausal breast cancer risk, opportunity remains for future research. To begin, several newly hypothesized evidence (short dashed arrows). Grey arrows relating biomarkers to each other are proposed in the literature; some of these relations are hypothesized, whereas others are well-established. Adapted from [11]

biologic pathways require further study to better understand their etiologic roles in postmenopausal breast cancer and hence, their suitability as biomarkers. Second, although our list of proposed biomarkers is fairly comprehensive, encompassing our own areas of research, additional biomarkers can be considered for our model, including, for example, those related to DNA repair mechanisms, additional epigenetic indicators, other adipokines, and anti-inflammatory cytokines. Several of these mechanisms may be more intensive to measure than what has been done previously, such as genomic alterations, and may require a tissue-specific approach. Hypothesized biomarkers also could be removed from the model as data becomes available; e.g., exercise-only trials in older women thus far have not produced changes in circulating adiponectin, TNF- $\alpha$, or IL- 6 levels. Third, biomarkerexercise RCTs could be analyzed using a systems epidemiology approach [109], quantifying the direct and indirect causal effects of exercise on multiple biomarkers simultaneously rather than on single biomarkers as in previous analyses. Results would identify pivotal exercise-induced biomarker changes and important mediators of those changes as targets 
for future intervention research. Fourth, new RCTs in healthy postmenopausal women are needed to compare different types of physical activity (e.g., aerobic versus resistance) and different doses (i.e., frequency, duration and intensity) to clarify the optimal prescription for breast cancer risk reduction. Studies exploring interindividual variability in exercise-induced biomarker changes, e.g., due to common genetic polymorphisms, would help tailor exercise prescriptions.

One recently completed, noteworthy study is the Breast Cancer and Exercise Trial in Alberta (BETA Trial). This trial, comprising 400 postmenopausal, previously inactive women who were randomized to undertake either 150 or 300 minutes per week of aerobic exercise for 1 year, was designed specifically to determine the optimal activity dose for lowering postmenopausal breast cancer risk. In secondary analyses of the ALPHA trial data, higher exercise duration was associated with desirable changes in adiposity and circulating sex steroid hormone levels, HOMA-IR, leptin, and CRP levels, with the greatest benefit observed for women undertaking $>150$ minutes per week [32] or $>225$ minutes per week $[50,70,110]$ of aerobic exercise. Therefore, previous biomarker-exercise RCTs that generated null findings in primary analyses may have been limited by the relatively low dose of exercise that was prescribed or attained by study participants. Furthermore, it is possible that the current physical activity guidelines recommended for cancer prevention are insufficient for postmenopausal breast cancer. A recent prospective investigation of 30,797 postmenopausal women [111•] found no significant association between invasive breast cancer incidence and near-achievement of the WCRF/AICR 2007 minimum physical activity recommendations [1] for cancer prevention.

\section{Conclusions}

Evidence from randomized exercise trials in healthy, overweight and obese postmenopausal women implies that moderate-vigorous aerobic exercise prescriptions of 150 225 minutes per week over 12 months can lower estradiol levels by approximately $5-10 \%$ on average, primarily through total body weight loss. Yet, there is biologic plausibility that some exercise-induced biomarker changes do not require loss of body fat, whereas others depend on abdominal fat loss. The preventive effect of exercise is probably the culmination of numerous interrelated biomarker changes that, when combined, act additively or synergistically to impede carcinogenesis in the breast. The level of physical activity required to induce these changes could be higher than the minimum level currently advised for cancer prevention and might depend on individual factors, e.g., genetic constitution. Identifying a physical activity prescription that produces clinically meaningful changes in key biomarkers and subgroups of women who would benefit the most from physical activity is a priority for future research. These recommendations will be used to inform prevention strategies for breast cancer after menopause.

Acknowledgments The authors thank Darren Brenner for reviewing our manuscript. Christine Friedenreich was funded by a Health Senior Scholar Award from Alberta Innovates-Health Solutions (AI-HS) and through the Alberta Cancer Foundation's Weekend to End Women's Cancers Breast Cancer Chair. Shannon Conroy was funded by Postdoctoral Fellowships from the Canadian Institutes for Health Research and AI-HS.

\section{Compliance with Ethics Guidelines}

Conflict of Interest Heather K. Neilson, Shannon M. Conroy, and Christine M. Friedenreich declare that they have no conflict of interest.

Human and Animal Rights and Informed Consent This article does not contain any studies with human or animal subjects performed by any of the authors.

Open Access This article is distributed under the terms of the Creative Commons Attribution License which permits any use, distribution, and reproduction in any medium, provided the original author(s) and the source are credited.

\section{References}

Papers of particular interest, published recently, have been highlighted as:

- Of importance

•. Of major importance

1. Food, Nutrition, Physical Activity, and the Prevention of Cancer: a Global Perspective. Washington, DC: World Cancer Research Fund and the American Institute for Cancer Research; 2007.

2. Renehan AG, Tyson M, Egger M, et al. Body-mass index and incidence of cancer: a systematic review and meta-analysis of prospective observational studies. Lancet. 2008;371(9612):569-78.

3. Suzuki R, Orsini N, Saji S, et al. Body weight and incidence of breast cancer defined by estrogen and progesterone receptor status: a meta-analysis. Int J Cancer. 2009;124(3):698-712.

4. Niraula S, Ocana A, Ennis M, Goodwin PJ. Body size and breast cancer prognosis in relation to hormone receptor and menopausal status: a meta-analysis. Breast Cancer Res Treat. 2012;134(2): 769-81.

5. Patterson RE, Cadmus LA, Emond JA, Pierce JP. Physical activity, diet, adiposity and female breast cancer prognosis: a review of the epidemiologic literature. Maturitas. 2010;66(1):5-15.

6. Ballard-Barbash R, Hunsberger S, Alciati MH, et al. Physical activity, weight control, and breast cancer risk and survival: clinical trial rationale and design considerations. J Natl Cancer Inst. 2009;101(9):630-43.

7. Kushi LH, Byers T, Doyle C, et al. American Cancer Society Guidelines on Nutrition and Physical Activity for Cancer Prevention: Reducing the Risk of Cancer With Healthy Food Choices and Physical Activity. CA Cancer J Clin. 2006;56(5): 254-81.

8. Lynch BM, Neilson HK, Friedenreich CM. Physical activity and breast cancer prevention. Recent Results Cancer Res. 2011;186: 
13-42. A comprehensive literature review that we conducted in December 2009, relating physical activity to breast cancer risk. Our currently proposed biologic model was updated from this earlier review.

9. Wu Y, Zhang D, Kang S. Physical activity and risk of breast cancer: a meta-analysis of prospective studies. Breast Cancer Res Treat. 2013;137(3):869-82.

10. Rundle A. Molecular epidemiology of physical activity and cancer. Cancer Epidemiol Biomarkers Prev. 2005;14(1):227-36.

11. Neilson HK, Friedenreich CM, Brockton NT, Millikan RC. Physical activity and postmenopausal breast cancer: proposed biologic mechanisms and areas for future research. Cancer Epidemiol Biomarkers Prev. 2009;18(1):11-27.

12. Ballard-Barbash R, Friedenreich CM, Courneya KS, et al. Physical activity, biomarkers, and disease outcomes in cancer survivors: a systematic review. J Natl Cancer Inst. 2012;104(11): 815-40. The effects of exercise in breast cancer survivors was beyond the scope of our review. However, this systematic review by Ballard-Barbash et al. is recommended for a review of exercise trials in cancer survivors.

13. Lof M, Bergstrom K, Weiderpass E. Physical activity and biomarkers in breast cancer survivors: A systematic review. Maturitas. 2012.

14. Patterson RE, Rock CL, Kerr J, et al. Metabolism and breast cancer risk: frontiers in research and practice. J Acad Nutr Diet. 2013;113(2):288-96. This article reviews hypothesized mechanisms mediating the relation between obesity and breast cancer risk/progression, with parallels to our own biologic model of physical activity effects. Unlike Patterson et al. our review focuses only on postmenopausal breast cancer risk.

15. Rose DP, Vona-Davis L. Biochemical and molecular mechanisms for the association between obesity, chronic Inflammation, and breast cancer. Biofactors. 2013. doi:10.1002/biof.1109.

16. John EM, Phipps AI, Sangaramoorthy M. Body size, modifying factors, and postmenopausal breast cancer risk in a multiethnic population: the San Francisco Bay Area Breast Cancer Study. SpringerPlus. 2013;2(1):239.

17. Liedtke S, Schmidt ME, Vrieling A, et al. Postmenopausal sex hormones in relation to body fat distribution. Obesity (Silver Spring). 2012;20(5):1088-95. Findings support the hypothesis that exercise interventions may need to target abdominal fat in order to reduce postmenopausal breast cancer risk.

18. Donnelly JE, Blair SN, Jakicic JM, et al. American College of Sports Medicine Position Stand Appropriate physical activity intervention strategies for weight loss and prevention of weight regain for adults. Med Sci Sports Exerc. 2009;41(2):459-71.

19. Lau DC, Douketis JD, Morrison KM, et al. 2006 Canadian clinical practice guidelines on the management and prevention of obesity in adults and children [summary]. CMAJ. 2007;176(8):S1-S13.

20. Sims ST, Larson JC, Lamonte MJ, et al. Physical activity and body mass: changes in younger versus older postmenopausal women. Med Sci Sports Exerc. 2012;44(1):89-97.

21. Slentz CA, Bateman LA, Willis LH, et al. Effects of aerobic vs. resistance training on visceral and liver fat stores, liver enzymes, and insulin resistance by HOMA in overweight adults from STRRIDE AT/RT. Am J Physiol Endocrinol Metab. 2011;301(5):E1033-9. This randomized trial in 155 men and women age 18-79 years suggested that aerobic exercise was more beneficial than resistance exercise for lowering central body fat and improving insulin sensitivity. A similar trial in postmenopausal women that examines additional biomarkers for breast cancer would be informative.

22. Friedenreich CM, Woolcott CG, McTiernan A, et al. Adiposity changes after a 1-year aerobic exercise intervention among postmenopausal women: a randomized controlled trial. Int J Obes (Lond). 2010;35:427-35.
23. Irwin ML, Yasui Y, Ulrich CM, et al. Effect of exercise on total and intra-abdominal body fat in postmenopausal women: a randomized controlled trial. JAMA. 2003;289(3):323-30.

24. Foster-Schubert KE, Alfano CM, Duggan CR, et al. Effect of diet and exercise, alone or combined, on weight and body composition in overweight-to-obese postmenopausal women. Obesity. 2012;20(8):1628-38. This article reports on adiposity changes in the Nutrition and Exercise in Women $(N E W)$ trial. The study population is directly relevant to postmenopausal breast cancer risk.

25. Velthuis MJ, Schuit AJ, Peeters PH, Monninkhof EM. Exercise program affects body composition but not weight in postmenopausal women. Menopause. 2009;16(4):777-84.

26. Bergstrom I, Lombardo C, Brinck J. Physical training decreases waist circumference in postmenopausal borderline overweight women. Acta Obstet Gynecol Scand. 2009;88(3):308-13.

27. Key T, Appleby P, Barnes I, Reeves G. Endogenous sex hormones and breast cancer in postmenopausal women: reanalysis of nine prospective studies. J Natl Cancer Inst. 2002;94(8):60616.

28. Chlebowski RT, Kuller LH, Prentice RL, et al. Breast cancer after use of estrogen plus progestin in postmenopausal women. N Engl J Med. 2009;360(6):573-87.

29. Uray IP, Brown PH. Prevention of breast cancer: current state of the science and future opportunities. Expert Opin Investig Drugs. 2006;15(12):1583-600.

30. Yager JD, Davidson NE. Estrogen carcinogenesis in breast cancer. N Engl J Med. 2006;354(3):270-82.

31. Copeland JL, Tremblay MS. Effect of HRT on hormone responses to resistance exercise in post-menopausal women. Maturitas. 2004;48(4):360-71.

32. Friedenreich CM, Woolcott CG, McTiernan A, et al. Alberta physical activity and breast cancer prevention trial: sex hormone changes in a year-long exercise intervention among postmenopausal women. J Clin Oncol. 2010;28(9):1458-66.

33. Yoo EJ, Jun TW, Hawkins S. The effects of a walking exercise program on fall-related fitness, bone metabolism, and fall-related psychological factors in elderly women. Res Sports Med. 2010;18(4):236-50.

34. Monninkhof EM, Velthuis MJ, Peeters PH, et al. Effect of exercise on postmenopausal sex hormone levels and role of body fat: a randomized controlled trial. J Clin Oncol. 2009;27(27):4492-9.

35. McTiernan A, Tworoger SS, Ulrich CM, et al. Effect of exercise on serum estrogens in postmenopausal women: a 12-month randomized clinical trial. Cancer Res. 2004;64(8):2923-8.

36.• Campbell KL, Foster-Schubert KE, Alfano CM, et al. Reducedcalorie dietary weight loss, exercise, and sex hormones in postmenopausal women: randomized controlled trial. J Clin Oncol. 2012;30(19):2314-26. This article reports on primary outcomes from the Nutrition and Exercise in Women (NEW) trial. This trial provides the strongest evidence to-date to distinguish the effects of exercise from those of weight loss on sex hormone levels in healthy postmenopausal women.

37. Figueroa A, Going SB, Milliken LA, et al. Effects of exercise training and hormone replacement therapy on lean and fat mass in postmenopausal women. J Gerontol A Biol Sci Med Sci. 2003;58(3):M266-70.

38. Kim JW, Kim DY. Effects of aerobic exercise training on serum sex hormone binding globulin, body fat index, and metabolic syndrome factors in obese postmenopausal women. Metab Syndr Relat Disord. 2012;10(6):452-7.

39. Orsatti FL, Nahas EA, Maesta N, et al. Plasma hormones, muscle mass and strength in resistance-trained postmenopausal women. Maturitas. 2008;59(4):394-404.

40. Atkinson C, Lampe JW, Tworoger SS, et al. Effects of a moderate intensity exercise intervention on estrogen metabolism in 
postmenopausal women. Cancer Epidemiol Biomarkers Prev. 2004;13(5):868-74.

41. Campbell KL, Foster-Schubert KE, Makar KW, et al. Gene Expression Changes in Adipose Tissue with Diet- and/or Exercise-Induced Weight Loss. Cancer Prev Res. 2013;6(3): 217-31.

42. McTiernan A, Tworoger SS, Rajan KB, et al. Effect of exercise on serum androgens in postmenopausal women: a 12-month randomized clinical trial. Cancer Epidemiol Biomarkers Prev. 2004;13(7): 1099-105.

43. Carpenter CL, Duvall K, Jardack P, et al. Weight loss reduces breast ductal fluid estrogens in obese postmenopausal women: a single arm intervention pilot study. Nutr J. 2012;11:102. This study demonstrated weight loss-associated decreased estrogens levels in the breast, whereas most other studies describe changes in circulating, whole-body estrogen levels.

44. Jones ME, Schoemaker M, Rae M, et al. Changes in estradiol and testosterone levels in postmenopausal women after changes in body mass index. J Clin Endocrinol Metab. 2013;98(7):296774.

45. Autier P, Koechlin A, Boniol M, et al. Serum insulin and Cpeptide concentration and breast cancer: a meta-analysis. Cancer Causes Control. 2013;24(5):873-83.

46. Ahern TP, Hankinson SE, Willett WC, et al. Plasma C-peptide, mammographic breast density, and risk of invasive breast cancer. Cancer Epidemiol Biomarkers Prev. 2013;22(10):1786-96.

47. Rose DP, Vona-Davis L. The cellular and molecular mechanisms by which insulin influences breast cancer risk and progression. Endocr Relat Cancer. 2012;19(6):R225-41.

48. Goodwin PJ, Stambolic V. Obesity and insulin resistance in breast cancer-chemoprevention strategies with a focus on metformin. Breast. 2011;20 Suppl 3:S31-5.

49. Colberg SR, Sigal RJ, Fernhall B, et al. Exercise and Type 2 Diabetes: The American College of Sports Medicine and the American Diabetes Association: joint position statement executive summary. Diabetes Care. 2010;33(12):2692-6.

50. Friedenreich CM, Neilson HK, Woolcott CG, et al. Changes in insulin resistance indicators, insulin-like growth factors, and adipokines in a year-long trial of aerobic exercise in postmenopausal women. Endocr Relat Cancer. 2011;18(3):357-69.

51. Frank LL, Sorensen BE, Yasui Y, et al. Effects of exercise on metabolic risk variables in overweight postmenopausal women: a randomized clinical trial. Obes Res. 2005;13(3):615-25.

52. Mason C, Foster-Schubert KE, Imayama I, et al. Dietary weight loss and exercise effects on insulin resistance in postmenopausal women. Am J Prev Med. 2011;41(4):366-75. This article reports on the Nutrition and Exercise in Women (NEW) trial. This trial provides the strongest evidence to-date to distinguish the effects of exercise from those of weight loss on insulin resistance indicators in a study population that is directly relevant to postmenopausal breast cancer.

53. Arsenault BJ, Cote M, Cartier A, et al. Effect of exercise training on cardiometabolic risk markers among sedentary, but metabolically healthy overweight or obese post-menopausal women with elevated blood pressure. Atherosclerosis. 2009;207(2):530-3.

54. Friedenreich CM, Neilson HK, Woolcott CG, et al. Mediators and moderators of the effects of a year-long exercise intervention on endogenous sex hormones in postmenopausal women. Cancer Causes Control. 2011;22(10):1365-73. A novel analytical approach is used to identify potential mediators of exercise-induced sex hormone changes. Formal definitions of mediators and moderators are drawn from the psychology literature.

55. Brochu M, Tchernof A, Dionne IJ, et al. What are the physical characteristics associated with a normal metabolic profile despite a high level of obesity in postmenopausal women? J Clin Endocrinol Metab. 2001;86(3):1020-5.
56. Tresierras MA, Balady GJ. Resistance training in the treatment of diabetes and obesity: mechanisms and outcomes. J Cardiopulm Rehabil Prev. 2009;29(2):67-75.

57. Figueroa A, Vicil F, Sanchez-Gonzalez MA, et al. Effects of diet and/or low-intensity resistance exercise training on arterial stiffness, adiposity, and lean mass in obese postmenopausal women. Am J Hypertens. 2013;26(3):416-23.

58. Waki H, Tontonoz P. Endocrine functions of adipose tissue. Annu Rev Pathol. 2007;2:31-56.

59. You T, Arsenis NC, Disanzo BL, LaMonte MJ. Effects of exercise training on chronic inflammation in obesity. Sports Med. 2013;43(4):243-56.

60. Jarde T, Perrier S, Vasson MP, Caldefie-Chezet F. Molecular mechanisms of leptin and adiponectin in breast cancer. Eur $\mathrm{J}$ Cancer. 2011;47(1):33-43.

61. He B, Niu J, Jiang L, et al. The association between leptin level and breast cancer: a meta-analysis. PLoS ONE. 2013;8(6):e67349.

62. Ollberding NJ, Kim Y, Shvetsov YB, et al. Prediagnostic Leptin, Adiponectin, C-Reactive Protein, and the Risk of Postmenopausal Breast Cancer. Cancer Prev Res. 2013;6(3):188-95.

63. Gross AL, Newschaffer CJ, Hoffman-Bolton J, et al. Adipocytokines, inflammation, and breast cancer risk in postmenopausal women: a prospective study. Cancer Epidemiol Biomarkers Prev. 2013;22(7):1319-24.

64. Singh S, Liu L-Y, Wang M, et al. The role of adiponectin in breast cancer: a meta-analysis. PLoS ONE. 2013;8(8):e73183.

65. Heikkila K, Harris R, Lowe G, et al. Associations of circulating Creactive protein and interleukin- 6 with cancer risk: findings from two prospective cohorts and a meta-analysis. Cancer Causes Control. 2009;20(1):15-26.

66. Shen C, Sun H, Sun D, et al. Polymorphisms of tumor necrosis factor-alpha and breast cancer risk: a meta-analysis. Breast Cancer Res Treat. 2010;126(3):763-70.

67. Abbenhardt C, McTiernan A, Alfano CM, et al. Effects of individual and combined dietary weight loss and exercise interventions in postmenopausal women on adiponectin and leptin levels. J Intern Med. 2013;274(2):163-75. This article reports on the Nutrition and Exercise in Women $(N E W)$ trial. This trial provides the strongest evidence to-date to distinguish the effects of exercise from those of weight loss on adiponectin and leptin levels in a study population that is directly relevant to postmenopausal breast cancer.

68. Imayama I, Ulrich CM, Alfano CM, et al. Effects of a caloric restriction weight loss diet and exercise on inflammatory biomarkers in overweight/obese postmenopausal women: a randomized controlled trial. Cancer Res. 2012;72(9):2314-26. This article reports on the Nutrition and Exercise in Women (NEW) trial. This trial provides the strongest evidence to-date to distinguish the effects of exercise from those of weight loss on inflammatory markers in a study population that is directly relevant to postmenopausal breast cancer.

69. Giannopoulou I, Fernhall B, Carhart R, et al. Effects of diet and/or exercise on the adipocytokine and inflammatory cytokine levels of postmenopausal women with type 2 diabetes. Metabolism. 2005;54(7):866-75.

70. Friedenreich CM, Neilson HK, Woolcott CG, et al. Inflammatory marker changes in a year-long randomized exercise intervention trial among postmenopausal women. Cancer Prev Res. 2012;5(1):98-108.

71. Campbell PT, Campbell KL, Wener MH, et al. A yearlong exercise intervention decreases CRP among obese postmenopausal women. Med Sci Sports Exerc. 2009;41(8):1533-9.

72. Stewart LK, Earnest CP, Blair SN, Church TS. Effects of different doses of physical activity on C-reactive protein among women. Med Sci Sports Exerc. 2010;42(4):701-7.

73. Phillips MD, Patrizi RM, Cheek DJ, et al. Resistance training reduces subclinical inflammation in obese, postmenopausal women. Med Sci Sports Exerc. 2012;44(11):2099-110. 
74. Hanahan D, Weinberg RA. Hallmarks of cancer: the next generation. Cell. 2011;144(5):646-74.

75. Standish LJ, Sweet ES, Novack J, et al. Breast cancer and the immune system. J Soc Integr Oncol. 2008;6(4):158-68.

76. Walsh NP, Gleeson M, Shephard RJ, et al. Position statement Part one: Immune function and exercise. ExercImmunol Rev. 2011;17: 6-63.

77. Simpson RJ, Lowder TW, Spielmann G, et al. Exercise and the aging immune system. Ageing Res Rev. 2012;11(3):404-20.

78. Haaland DA, Sabljic TF, Baribeau DA, et al. Is regular exercise a friend or foe of the aging immune system? A systematic review. Clin J Sport Med. 2008;18(6):539-48.

79. Campbell PT, Wener MH, Sorensen B, et al. Effect of exercise on in vitro immune function: a 12-month randomized, controlled trial among postmenopausal women. J Appl Physiol. 2008;104(6): $1648-55$.

80. Vera-Ramirez L, Sanchez-Rovira P, Ramirez-Tortosa MC, et al. Free radicals in breast carcinogenesis, breast cancer progression and cancer stem cells Biological bases to develop oxidative-based therapies. Crit Rev Oncol Hematol. 2011;80(3):347-68.

81. Crujeiras AB, Diaz-Lagares A, Carreira MC, et al. Oxidative stress associated to dysfunctional adipose tissue: a potential link between obesity, type 2 diabetes mellitus and breast cancer. Free Radic Res. 2013;47(4):243-56.

82. Ziech D, Franco R, Pappa A, Panayiotidis MI. Reactive oxygen species (ROS)-induced genetic and epigenetic alterations in human carcinogenesis. Mutat Res. 2011;711(1-2):167-73.

83. Prescott J, Wentzensen IM, Savage SA, De Vivo I. Epidemiologic evidence for a role of telomere dysfunction in cancer etiology. Mutat Res. 2012;730(1-2):75-84.

84. Radak Z, Chung HY, Goto S. Systemic adaptation to oxidative challenge induced by regular exercise. Free Radic Biol Med. 2008;44(2):153-9.

85. Campbell PT, Gross MD, Potter JD, et al. Effect of exercise on oxidative stress: a 12-month randomized, controlled trial. Med Sci Sports Exerc. 2010;42(8):1448-53.

86. Qian G, Xue K, Tang L, et al. Mitigation of oxidative damage by green tea polyphenols and Tai Chi exercise in postmenopausal women with osteopenia. PLoS One. 2012;7(10):e48090.

87. Vincent HK, Bourguignon C, Vincent KR. Resistance training lowers exercise-induced oxidative stress and homocysteine levels in overweight and obese older adults. Obesity (Silver Spring). 2006;14(11):1921-30.

88. Mason C, Risques R-A, Xiao L, et al. Independent and combined effects of dietary weight loss and exercise on leukocyte telomere length in postmenopausal women. Obesity. 2013. doi:10.1002/ oby.20509. This article reports on the Nutrition and Exercise in Women (NEW) trial. This article presents novel findings surrounding the effects of exercise-alone versus weight loss on leukocytle telomere length in a study population that is directly relevant to postmenopausal breast cancer.

89. Hovatta I, de Mello VD, Kananen L, et al. Leukocyte telomere length in the Finnish Diabetes Prevention Study. PLoS ONE. 2012;7(4): 34948

90. Woo HD, Kim J. Global DNA hypomethylation in peripheral blood leukocytes as a biomarker for cancer risk: a meta-analysis. PLoS One. 2012;7(4):e34615.

91. Delgado-Cruzata L, Wu HC, Perrin M, et al. Global DNA methylation levels in white blood cell DNA from sisters discordant for breast cancer from the New York site of the Breast Cancer Family Registry. Epigenetics. 2012;7(8):868-74.

92. Deroo LA, Bolick SC, Xu Z et al. Global DNA methylation and one-carbon metabolism gene polymorphisms and the risk of breast cancer in the Sister Study. Carcinogenesis. 2013

93. Ntanasis-Stathopoulos J, Tzanninis JG, Philippou A, Koutsilieris M. Epigenetic regulation on gene expression induced by physical exercise. J Musculoskelet Neuronal Interact. 2013;13(2):133-46. While our review focuses on global DNA hypomethylation as a potentially modifiable epigenetic biomarker of breast cancer risk, this article comprehensively reviews a variety of epigenetic changes induced by exercise. Mechanisms related to carcinogenesis, aging, neurophysiological disorders, metabolic syndrome, and cardiovascular disease are described.

94. Yang AS, Estecio MR, Doshi K, et al. A simple method for estimating global DNA methylation using bisulfite PCR of repetitive DNA elements. Nucleic Acids Res. 2004;32(3):e38.

95. White AJ, Sandler DP, Bolick SCE, et al. Recreational and household physical activity at different time points and DNA global methylation. Eur J Cancer. 2013;49(9):2199-206.

96. Zhang FF, Cardarelli R, Carroll J, et al. Physical activity and global genomic DNA methylation in a cancer-free population. Epigenetics. 2011;6(3):293-9.

97. Bauer SR, Hankinson SE, Bertone-Johnson ER, Ding EL. Plasma vitamin D levels, menopause, and risk of breast cancer: doseresponse meta-analysis of prospective studies. Medicine (Baltimore). 2013;92(3):123-31.

98. Wang D, Velez de-la-Paz OI, Zhai JX, Liu DW. Serum 25hydroxyvitamin $\mathrm{D}$ and breast cancer risk: a meta-analysis of prospective studies. Tumour Biol. 2013.

99. Wortsman J, Matsuoka LY, Chen TC, et al. Decreased bioavailability of vitamin D in obesity. Am J Clin Nutr. 2000;72(3):690-3.

100. Mason C, Xiao L, Imayama I, et al. Effects of weight loss on serum vitamin D in postmenopausal women. Am J Clin Nutr. 2011;94(1):95-103. This article reports on the Nutrition and Exercise in Women (NEW) trial. This trial provides strong evidence to support the hypothesis that serum 25-hydroxyvitamin D levels are mediated by body fat in postmenopausal women.

101. Rock CL, Emond JA, Flatt SW, et al. Weight loss is associated with increased serum 25-hydroxyvitamin D in overweight or obese women. Obesity. 2012;20(11):2296-301.

102. Tamimi RM, Byrne C, Colditz GA, Hankinson SE. Endogenous hormone levels, mammographic density, and subsequent risk of breast cancer in postmenopausal women. J Natl Cancer Inst. 2007;99(15):1178-87.

103. Yaghjyan L, Colditz GA, Wolin K. Physical activity and mammographic breast density: a systematic review. Breast Cancer Res Treat. 2012;135(2):367-80.

104. Key TJ, Appleby PN, Reeves GK, Roddam AW. Insulin-like growth factor 1 (IGF1), IGF binding protein 3 (IGFBP3), and breast cancer risk: pooled individual data analysis of 17 prospective studies. Lancet Oncol. 2010;11(6):530-42.

105. McTiernan A, Sorensen B, Yasui Y, et al. No effect of exercise on insulin-like growth factor 1 and insulin-like growth factor binding protein 3 in postmenopausal women: a 12-month randomized clinical trial. Cancer Epidemiol Biomarkers Prev. 2005;14(4):1020-1.

106. Mason C, Xiao L, Duggan C, et al. Effects of Dietary Weight Loss and Exercise on Insulin-Like Growth Factor-I and Insulin-Like Growth Factor-Binding Protein-3 in Postmenopausal Women: A Randomized Controlled Trial. Cancer Epidemiol Biomarkers Prev. 2013;22(8):1457-63. This article provides strong evidence from the four-armed Nutrition and Exercise in Women (NEW) trial that IGF-1 bioavailability is an unlikely mechanism through which weight loss mediates postmenopausal breast cancer risk, which refutes common theories surrounding IGF-1.

107. Liedtke S, Schmidt ME, Becker S, et al. Physical activity and endogenous sex hormones in postmenopausal women: to what extent are observed associations confounded or modified by BMI? Cancer Causes Control. 2011;22(1):81-9.

108.• van Gemert WAM, Iestra JI, Schuit AJ, et al. Design of the SHAPE-2 study: the effect of physical activity, in addition 
to weight loss, on biomarkers of postmenopausal breast cancer risk. BMC Cancer. 2013;13(1):395. Results from the SHAPE-2 trial will help discern whether or not exercise contibutes to biomarker changes independently of weight loss in healthy postmenopausal women.

109. Joffe M, Gambhir M, Chadeau-Hyam M, Vineis P. Causal diagrams in systems epidemiology. Emerg Themes Epidemiol. 2012;9(1):1.

110. Friedenreich CM, Woolcott CG, McTiernan A, et al. Adiposity changes after a 1-year aerobic exercise intervention among postmenopausal women: a randomized controlled trial. Int $\mathrm{J}$ Obes (Lond). 2011;35(3):427-35.

111. Hastert TA, Beresford SAA, Patterson RE, et al. Adherence to WCRF/AICR Cancer Prevention Recommendations and Risk of Postmenopausal Breast Cancer. Cancer Epidemiol Biomarkers Prev. 2013;22(9):1498-508. Findings from this prospective study of 30,797 postmenopausal women in the U.S. imply that nearachievement of the minimum physical activity recommendation for cancer prevention does not significantly decrease the risk of postmenopausal invasive breast cancer. 